\title{
Explicit finite-difference solution of two-dimensional solute transport with periodic flow in homogenous porous media
}

\author{
Alexandar Djordjevich ${ }^{1}$, Svetislav Savović ${ }^{1,2^{*}}$, Aco Janićijević ${ }^{3}$ \\ ${ }^{1}$ City University of Hong Kong, 83 Tat Chee Avenue, Kowloon, Hong Kong, China. \\ ${ }^{2}$ University of Kragujevac, Faculty of Science, R. Domanovića 12, 34000 Kragujevac, Serbia. \\ ${ }^{3}$ Faculty of Technology and Metallurgy, University of Belgrade, Belgrade, Serbia. \\ ${ }^{*}$ Corresponding author. Fax: +381-34-335040. E-mail: savovic@kg.ac.rs
}

\begin{abstract}
The two-dimensional advection-diffusion equation with variable coefficients is solved by the explicit finitedifference method for the transport of solutes through a homogenous two-dimensional domain that is finite and porous. Retardation by adsorption, periodic seepage velocity, and a dispersion coefficient proportional to this velocity are permitted. The transport is from a pulse-type point source (that ceases after a period of activity). Included are the firstorder decay and zero-order production parameters proportional to the seepage velocity, and periodic boundary conditions at the origin and at the end of the domain. Results agree well with analytical solutions that were reported in the literature for special cases. It is shown that the solute concentration profile is influenced strongly by periodic velocity fluctuations. Solutions for a variety of combinations of unsteadiness of the coefficients in the advection-diffusion equation are obtainable as particular cases of the one demonstrated here. This further attests to the effectiveness of the explicit finite difference method for solving two-dimensional advection-diffusion equation with variable coefficients in finite media, which is especially important when arbitrary initial and boundary conditions are required.
\end{abstract}

Keywords: Two dimensional advection-diffusion equation; Mass transfer; Finite difference method.

\section{INTRODUCTION}

Environmental concerns have inspired much research interest in solute transport through porous media. Factors affecting this transport include the solvent and solute properties, fluid velocity field within the porous medium, and micro-geometry such as the shape, size, and location of the solid part of the medium or the layout of the voids. Advective-dispersive phenomena often dominate the process (Dehghan, 2004; Dhawan et al., 2012). Thus for example, contaminants of groundwater permeate through pores in the ground and adsorption attenuates the pollution concentration as the pollutants adhere to the solid surface.

The partial differential advection-diffusion equation (ADE) describes water transfer in soils (Parlange, 1980), dispersion of tracers in porous media (Fattah and Hoopes, 1985), the spread of pollutants in rivers and streams (Chatwin and Allen, 1985), the dispersion of dissolved material in estuaries and coastal seas (Holly and Usseglio-Polatera, 1984), contaminant dispersion in shallow lakes (Salmon et al., 1980), the absorption of chemicals into beds (Lapidus and Amundston, 1952), long-range transport of pollutants in the atmosphere (Zlatev et al., 1984), forced cooling by fluids of solid material such as windings in turbo generators (Gane and Stephenson, 1979), thermal pollution in river systems (Chaudhry et al., 1983), flow in porous media (Kumar, 1988) and dispersion of dissolved salts in groundwater (Guvanasen and Volker, 1983).

Logan and Zlotnik (1995) proposed analytical solutions with a decay term for periodic input conditions through a semiinfinite domain to address fluctuations of the groundwater table and flow patterns caused by the periodicity of the sea level. Townley (1995) obtained analytical solutions based on the use of complex algebra for a periodic aquifer flow. Analytical solutions for one-dimensional ADE with temporally and spatially dependent dispersion problems are obtained by Jaiswal et al. (2009), Jaiswal et al. (2011), Kumar et al. (2010) and Yadav et al. (2011) using a Laplace transform technique. Chen and Liu (2011) reported a generalized analytical solution using a Laplace transform technique for advection-dispersion equation in finite spatial domain with arbitrary time-dependent inlet boundary conditions. A computer software package STANMOD which consists of a collection of analytical solutions of the advection-dispersion equation for transport problems has also been developed (Šimunek et al., 1999; van Genuchten et al., 2012).

Numerical solutions of the ADE have been reported for problems not covered by analytical solutions. For example, Zhao and Valliappan (1994) used a finite/infinite element technique, Dehghan (2004) used weighted finite difference technique, Karahan (2006) employed implicit finite difference techniques, Huang et al. (2008) used a finite element method, Ciftci et al. (2012) employed a meshless method, Nazir et al. (2016) used a new cubic trigonometric B-splines approach, Gharehbaghi (2017) employed a third- and fifth-order finite volume schemes and Kaya and Gharehbaghi (2014) used a various numerical methods. Notable numerical techniques are finite difference methods (FDM), finite element methods (FEM) and finite volume methods (FVM) - naturally, all three discretize governing equations and initial and boundary conditions.

For ADE with both constant and variable coefficients, Ahmed (2012) proposed an FDM discretization scheme that combined the Siemieniuch-Gradwell's approximation (Siemieniuch and Gladwell, 1978) for time and Dehghan's approximation (Dehghan, 2004) for the spatial variable. Savović and Djordjevich (2012) solved the ADE with variable coefficients in semi-infinite domain by an explicit formulation of the finite difference method (EFDM). Appadu (2013) applied three schemes (i.e., the Lax-Wendroff scheme, the Crank-Nicolson scheme, and the nonstandard finite difference scheme) to solve the ADE with constant coefficients in one-dimensional space. Gharehbaghi (2016) assessed the explicit and implicit forms of the differential quadrature method for time-dependent ADE 
with variable coefficients in one-dimensional semi-infinite domain.

A significant transverse solute transport has been noted even for very low longitudinal velocity and dispersivity relative to the respective values in the longitudinal direction (Yadav et al., 2012). Because one-dimensional models cannot capture such transverse transport, a two-dimensional model is required. Moreover, for different combinations of unsteadiness of the coefficients in the ADE, solutions can be obtained as particular cases of the one obtained in the present study.

Analytical solutions of the ADE have been reported for specific initial and boundary conditions. This lack of generality limits their applicability. Moreover, such solutions tend to be complex. Thus, analytical solutions of the ADE are usually lengthy and contain infinite series (Yadav et al., 2012). In contrast, numerical methods are generally flexible to accommodate arbitrary initial distribution and boundary conditions (Djordjevich and Savović, 2013; Savović and Caldwell, 2003; Savović and Caldwell, 2009). Being often unconditionally stable, implicit finite difference method (IFDM) allows larger step lengths than EFDM. Nevertheless, this does not translate into IFDM's higher computational efficiency because extremely large matrices must be manipulated at each calculation step. The EFDM algorithm is also simpler in addition to being more efficient computationally. We demonstrated earlier (Djordjevich and Savović, 2013; Savović and Djordjevich, 2012; Savović and Djordjevich, 2013) that the EFDM is effective and accurate in solving one-dimensional ADE with variable coefficients, as well as in solving two-dimensional ADE for solute transport from a pulse-type source. We propose in this paper (to the best of our knowledge for the first time) an effective, accurate and most simple explicit finite difference scheme for solving time-dependent ADEs in two-dimensional space. This allows us to derive a numerical solution for transport of solutes from a point source through a finite homogeneous and porous two-dimensional domain with the mixed-type boundary condition at the origin of the domain and with a periodic boundary condition at the end of the domain. The solution permits (i) a periodic seepage velocity and (ii) a dispersion coefficient that is proportional to the seepage velocity. The retardation process that occurs in the porous medium due to adsorption is also taken into account and the solute movement and dispersion are assumed in opposite directions (Al-Niami and Rushton, 1977; Marino, 1978). The illustrations of results obtained with the solution demonstrate solute transport in both longitudinal and transverse directions.

\section{ADVECTION-DIFFUSION EQUATION}

Let -the polluting solute particles enter a porous medium at a fixed location, continuously and at a constant rate up to a certain moment when the flow ceases (pulse-type source). The $\mathrm{ADE}$ in two-dimensional horizontal plane medium may be written as (Yadav et al., 2012):

$$
\begin{aligned}
& R_{f} \frac{\partial C(x, y, t)}{\partial t}=\frac{\partial}{\partial x}\left(D_{x}(x, t) \frac{\partial C(x, y, t)}{\partial x}-u(x, t) C(x, y, t)\right) \\
& +\frac{\partial}{\partial y}\left(D_{y}(y, t) \frac{\partial C(x, y, t)}{\partial y}-v(y, t) C(x, y, t)\right) \\
& -\gamma(x, y, t) C+\mu(x, y, t)
\end{aligned}
$$

where $C(x, y, t)$ is the dispersing solute concentration at a position $(x, y)$ at time $t ; D_{x}(\mathrm{x}, t)$ and $D_{y}(y, t)$ are dispersion coeffi- cients in directions $x$ and $y$, respectively; $u(x, t)$ and $v(y, t)$ are velocity coefficients in directions $x$ and $y$, respectively; $\gamma$ is the first-order decay coefficient and $\mu$ is the zero-order production coefficient. The retardation factor $R_{f}=\left(1+\frac{1-n_{p}}{n_{p}} K_{1}\right)$ accounts for the equilibrium linear adsorption process, where $n_{p}$ is the porosity of the medium and $K_{1}$ is an empirical constant (Cherry et al., 1984; Lapidus and Amundson, 1952).

The expressions for velocity components are assumed as (Yadav et al., 2012):

$u(x, t)=u_{0}|\sin (m t)| ; \quad v(y, t)=v_{0}|\sin (m t)|$

where $u_{0}$ and $v_{0}$ are uniform longitudinal and transverse velocity components, respectively, each of dimension $\left(L T^{-1}\right)$. The coefficient $m$ represents the unsteadiness parameter; it is in $T^{-1}$. The inclusion of transverse diffusion makes the dispersion problem two-dimensional. Hence, we consider dispersion coefficients as:

$$
D(x, t)=D_{x_{0}}|\sin (m t)|, \quad D(y, t)=D_{y_{0}}|\sin (m t)|
$$

where $D_{x_{0}}$ and $D_{y_{0}}$ are the initial longitudinal and transverse dispersion coefficients, respectively, each of dimension $\left(L^{2} T^{-1}\right)$. Furthermore, first-order decay and zero-order production are considered directly proportional to the seepage velocity and, thus, it is:

$$
\gamma=\gamma_{0}|\sin (m t)|, \quad \mu=\mu_{0}|\sin (m t)|
$$

where $\gamma_{0}$ and $\mu_{0}$ are constants of dimension $T^{-1}$ and $M L^{-3} T^{-1}$, respectively. Hence, (1) can now be rewritten as:

$$
\begin{aligned}
& R_{f} \frac{\partial C(x, y, t)}{\partial t}= \\
& \frac{\partial}{\partial x}\left(D_{x_{0}}|\sin (m t)| \frac{\partial C(x, y, t)}{\partial x}-u_{0}|\sin (m t)| C(x, y, t)\right) \\
& +\frac{\partial}{\partial y}\left(D_{y_{0}}|\sin (m t)| \frac{\partial C(x, y, t)}{\partial y}-v_{0}|\sin (m t)| C(x, y, t)\right) \\
& -\gamma_{0}|\sin (m t)| C+\mu_{0}|\sin (m t)|
\end{aligned}
$$

For the plane and horizontal porous medium, the initial solute concentration is set to vary with position according to (6) whereby $x$-axis is longitudinal and $y$-axis is transverse to the flow. The solute transport is in finite space $L_{x_{1}} \leq x \leq L_{x}$ and $L_{y_{1}} \leq y \leq L_{y}$ in the $x>0$ and $y>0$ directions. The source of the pollution is considered a uniform pulse at point $\left(L_{x}, L_{y}\right)$. Let the time of elimination of the point source be $t_{0}$. The initial and boundary conditions are (Yadav et al., 2012):

$$
\begin{aligned}
& C(x, y, t)=C_{i n} \exp (\alpha x+\alpha y), \quad L_{x_{1}} \leq x \leq L_{x} ; \\
& L_{y_{1}} \leq y \leq L_{y} ; t=0 \\
& C(x, y, t)=\left\{\begin{array}{lr}
C_{0}(1+\cos (m t)), & 0<t \leq t_{0} ; x=L_{x}, y=L_{y} \\
0, & t>t_{0} ; \quad x=L_{x}, y=L_{y}
\end{array}\right.
\end{aligned}
$$




$$
\frac{\partial C(x, y, t)}{\partial x}=\frac{u(x, t)}{2 D_{x}(x, t)} C(x, y, t), \quad x=L_{x_{1}} ; \frac{\partial C(x, y, t)}{\partial y}=\frac{v(y, t)}{2 D_{y}(y, t)} C(x, y, t), \quad y \rightarrow L_{y_{1}} ;(t \geq 0)
$$

where $C_{0}$ is the reference concentration representing the input concentration that is released uniformly by the source and $\alpha$ is a constant whose dimension is the inverse of length.

\section{ANALYTICAL SOLUTION OF ADVECTION-DIFFUSION EQUATION}

Analytical solution of the ADE (1), subject to initial condition (6) and boundary conditions (7) and (8), is (Yadav et al., 2012):

$$
\begin{aligned}
& C(\eta, T)=F_{1}(\eta, T)+C_{i n} F_{2}(\eta, T)+\left(2 C_{0}-\frac{\mu_{0}}{\gamma_{0}}\right) F_{3}(\eta, T)+C_{0} F_{4}(\eta, T) ; 0<T \leq T_{0} \\
& C(\eta, T)=F_{1}(\eta, T)+C_{i n} F_{2}(\eta, T)+C_{0}\left[2 F_{3}(\eta, T)-\left(2-m R_{f} T_{0}\right) F_{3}\left(\eta, T-T_{0}\right)\right]+C_{0}\left[F_{4}(\eta, T)-F_{4}\left(\eta, T-T_{0}\right)\right] ; T>T_{0}
\end{aligned}
$$

where

$$
\begin{aligned}
& F_{1}(\eta, T)=\frac{\mu_{0}}{\gamma_{0}}-\frac{\mu_{0}}{\gamma_{0}} \exp \left(-\gamma_{0} T\right)+\frac{\mu_{0}}{\gamma_{0}} \exp (-\beta L+\beta \eta)\left[\exp \left(-\gamma_{0} T\right) \frac{\cosh (\eta-L) \beta}{\cosh \left(L-L_{1}\right) \beta}-2 \pi D_{0} \exp \left(-\delta^{2} T\right) \sum_{n=0}^{\infty} E_{1} E_{2}\right] \\
& -\frac{\mu_{0}}{\gamma_{0}} \beta \exp \left(-\beta L_{1}+\beta \eta\right)\left\{\frac{\exp \left(-\gamma_{0} T\right)}{\beta} \frac{\sinh (\eta-L) \beta}{\cosh \left(L-L_{1}\right) \beta}-2\left(L-L_{1}\right) D_{0} \exp \left(-\delta^{2} T\right) \sum_{n=0}^{\infty}(-1)^{-n} \sin \left[(n+1 / 2) \pi \frac{(\eta-L)}{\left(L-L_{1}\right)}\right] E_{2}\right\} \\
& +\frac{\mu_{0}}{\gamma_{0}} \beta \exp \left(-\beta L_{1}+\beta \eta\right) \times\left\{E_{3}-2\left(L-L_{1}\right) D_{0} \exp \left(-\delta^{2} T\right) \sum_{n=0}^{\infty}(-1)^{-n} \sin \left[(n+1 / 2) \pi \frac{(\eta-L)}{\left(L-L_{1}\right)}\right] E_{4}\right\} \\
& F_{2}(\eta, T)=\exp \left[\alpha \eta+\left(b-\delta^{2}\right) T\right]-\exp \left[\alpha L-\beta L+\beta \eta-\delta^{2} T\right] \times\left[\exp (b T) \frac{\cosh \left(\eta-L_{1}\right) \sqrt{b / D_{0}}}{\cosh \left(L-L_{1}\right) \sqrt{b / D_{0}}}-2 \pi D_{0} \sum_{n=0}^{\infty} E_{1} E_{5}\right] \\
& -(\alpha-\beta) \exp \left[\alpha L_{1}-\beta L_{1}+\beta \eta-\delta^{2} T\right] \times\left\{\frac{\exp (b T)}{\sqrt{b / D_{0}}} \frac{\sinh (\eta-L) \sqrt{b / D_{0}}}{\cosh \left(L-L_{1}\right) \sqrt{b / D_{0}}}-2\left(L-L_{1}\right) D_{0} \sum_{n=0}^{\infty}(-1)^{-n} \sin \left[(n+1 / 2) \pi \frac{(\eta-L)}{\left(L-L_{1}\right)}\right] E_{5}\right\} \\
& F_{3}(\eta, T)=\exp (-\beta L+\beta \eta) E_{6}-2 \pi D_{0} \exp \left[(-\beta L+\beta \eta)-\delta^{2} T\right] \sum_{n=0}^{\infty} E_{1} E_{4} \\
& F_{4}(\eta, T)=\exp (-\beta L+\beta \eta)\left[\left\{\frac{\left(L-L_{1}\right)}{2} \sqrt{\frac{1}{\delta^{2} D_{0}}} \tanh \left(\left(L-L_{1}\right) \sqrt{\frac{\delta^{2}}{D_{0}}}\right)-m R_{f} T\right\} E_{6}-\frac{m R_{f}\left(\eta-L_{1}\right)}{2} E_{3}\right] \\
& -2 \pi D_{0} \exp \left[(-\beta L+\beta \eta)-\delta^{2} T\right] \sum_{n=1}^{\infty} \frac{m R_{f}\left(L-L_{1}\right)^{2} E_{1} E_{4}}{\left[(n+1 / 2)^{2} \pi^{2} D_{0}+\delta^{2}\left(L-L_{1}\right)^{2}\right]} \\
& E_{1}=(-1)^{-n}(n+1 / 2) \cos \left[(n+1 / 2) \pi \frac{\left(\eta-L_{1}\right)}{\left(L-L_{1}\right)}\right], E_{2}=\frac{\exp \left[-\frac{(n+1 / 2)^{2} \pi^{2} D_{0}}{\left(L-L_{1}\right)^{2}} T\right]}{\left[(n+1 / 2)^{2} \pi^{2} D_{0}+\omega^{2}\left(L-L_{1}\right)^{2}\right]}, E_{3}=\sqrt{\frac{1}{\delta^{2} D_{0}}} \frac{\sinh (\eta-L) \sqrt{\delta^{2} / D_{0}}}{\cosh \left(L-L_{1}\right) \sqrt{\delta^{2} / D_{0}}}, \\
& E_{4}=\frac{\exp \left(-\frac{(n+1 / 2)^{2} \pi^{2} D_{0}}{\left(L-L_{1}\right)^{2}} T\right)}{\left[(n+1 / 2)^{2} \pi^{2} D_{0}+\delta^{2}\left(L-L_{1}\right)^{2}\right]}, E_{5}=\frac{\exp \left[-\frac{(n+1 / 2)^{2} \pi^{2} D_{0}}{\left(L-L_{1}\right)^{2}} T\right]}{\left[(n+1 / 2)^{2} \pi^{2} D_{0}+b\left(L-L_{1}\right)^{2}\right]}, E_{6}=\frac{\cosh \left(\eta-L_{1}\right) \sqrt{\delta^{2} / D_{0}}}{\cosh \left(L-L_{1}\right) \sqrt{\delta^{2} / D_{0}}}, \\
& b=D_{0}(\alpha-\beta)^{2}, \quad \delta^{2}=\gamma_{0}+\omega^{2}, \quad \beta=\frac{U_{0}}{2 D_{0}}, \quad \omega^{2}=\frac{U_{0}^{2}}{4 D_{0}}, \quad D_{0}=D_{x_{0}}+D_{y_{0}}, \\
& U_{0}=u_{0}+v_{0}, \quad L=L_{x}+L_{y}, \quad L_{1}=L_{x_{1}}+L_{y_{1}}, \quad \eta=x+y, \quad T=\frac{1}{m R_{f}}[1-\cos (m t)] .
\end{aligned}
$$




\section{NUMERICAL METHOD}

In order to employ the EFDM to solve Equation (5), this equation is first rewritten in the following form:

$$
\begin{aligned}
& R_{f} \frac{\partial C(x, y, t)}{\partial t}=D_{x_{0}}|\sin (m t)| \frac{\partial C^{2}(x, y, t)}{\partial x^{2}} \\
& -u_{0}|\sin (m t)| \frac{\partial C(x, y, t)}{\partial x}+D_{y_{0}}|\sin (m t)| \frac{\partial C^{2}(x, y, t)}{\partial y^{2}} \\
& -v_{0}|\sin (m t)| \frac{\partial C(x, y, t)}{\partial y}-\gamma_{0}|\sin (m t)| C+\mu_{0}|\sin (m t)|
\end{aligned}
$$

The central difference scheme is used to represent the terms $\left(\partial^{2} C(x, t) / \partial x^{2}\right), \quad\left(\partial^{2} C(y, t) / \partial y^{2}\right), \quad(\partial C(x, t) / \partial x), \quad$ and $(\partial C(y, t) / \partial y)$ and a forward difference scheme is used for the derivative term $(\partial C(x, t) / \partial t)$ (Anderson, 1995). With these substitutions, Equation (12) transforms into:

$$
\begin{aligned}
& C_{i, j, k+1}=\left(G_{j}-E_{j}\right) C_{i+1, j, k}+\left(G_{j}+E_{j}\right) C_{i-1, j, k} \\
& +\left(H_{j}-F_{j}\right) C_{i, j+1, k}+\left(F_{j}+H_{j}\right) C_{i, j-1, k} \\
& +\left(1-I_{j}-2 H_{j}-2 G_{j}\right) C_{i, j, k}+J_{j}
\end{aligned}
$$

where indexes $i, j$ and $k$ refer to the discrete step lengths $\Delta x$, $\Delta y$, and $\Delta t$ for the coordinate $x$, coordinate $y$, and time $t$, respectively, and where:

$$
\begin{aligned}
E_{j}=\frac{u_{0}\left|\sin \left(m t_{j}\right)\right| \Delta t}{2 R_{f} \Delta x}, & F_{j}=\frac{v_{0}\left|\sin \left(m t_{j}\right)\right| \Delta t}{2 R_{f} \Delta y}, \\
G_{j}=\frac{D_{x_{0}}\left|\sin \left(m t_{j}\right)\right| \Delta t}{R_{f} \Delta x^{2}}, & H_{j}=\frac{D_{y_{0}}\left|\sin \left(m t_{j}\right)\right| \Delta t}{R_{f} \Delta y^{2}}, \\
I_{j}=\frac{\gamma_{0}\left|\sin \left(m t_{j}\right)\right| \Delta t}{R_{f}}, & J_{j}=\frac{\mu_{0}\left|\sin \left(m t_{j}\right)\right| \Delta t}{R_{f}}
\end{aligned}
$$

The truncation error for the difference Equation (13) is $O\left(\Delta t, \Delta x^{2}, \Delta y^{2}\right)$. Using a small-enough value of $\Delta x, \Delta y$, and $\Delta t$, the truncation error can be reduced until the accuracy achieved is within the error tolerance.

The initial condition (6) for Equation (13) is expressed in the finite difference form as:

$$
C_{i, j, 0}=C_{i n} \exp \left(\alpha x_{i}+\alpha y_{j}\right), \quad L_{x_{1}} \leq x_{i} \leq L_{x} ; L_{y_{1}} \leq y_{j} \leq L_{y} ; \quad t=0
$$

Boundary conditions (7) and (8), rewritten in the finite difference form, are:

$$
C_{N, R, k}=\left\{\begin{array}{rr}
C_{0}\left(1+\cos \left(m t_{k}\right)\right), & 0<t_{k} \leq t_{0} ; x_{N}=L_{x}, y_{R}=L_{y} \\
0, & t_{k}>t_{0} ; \quad x_{N}=L_{x}, y_{R}=L_{y}
\end{array}\right.
$$

$$
\begin{aligned}
& \frac{C_{1, j, k}-C_{0, j, k}}{\Delta x}=\frac{u_{0}}{2 D_{x_{0}}} C_{0, j, k}, \quad x=L_{x_{1}} ;(t \geq 0) \\
& \frac{C_{i, 1, k}-C_{i, 0, k}}{\Delta y}=\frac{v_{0}}{2 D_{y_{0}}} C_{i, 0, k}, \quad y=L_{y_{1}} ;(t \geq 0)
\end{aligned}
$$

where $N=\left(L_{x}-L_{x_{1}}\right) / \Delta x$ and $R=\left(L_{y}-L_{y_{1}}\right) / \Delta y$ are the grid dimensions in the $x$ and $y$ directions, respectively.

\section{NUMERICAL AND ANALYTICAL RESULTS}

Numerical solution of Eq. (13) is obtained by EFDM over a finite domain bounded by $0 \leq x \leq 1 \mathrm{~km}$ in the longitudinal and $0 \leq y \leq 1 \mathrm{~km}$ in the transverse direction. To enable the comparison of results, this was done for the same set of input data previously used by Yadav et al. (2012). The input parameters are $D_{x_{0}}=1.77 \mathrm{~km}^{2} /$ day, $D_{y_{0}}=0.177 \mathrm{~km}^{2} /$ day, $u_{0}=0.70 \mathrm{~km} /$ day, $v_{0}=0.070 \mathrm{~km} /$ day, $\alpha=0.025 \mathrm{~km}^{-1}, \gamma_{0}=0.01 \mathrm{day}^{-1}, \mu_{0}=0.01$, $C_{0}=1.0, C_{i n}=0.1$ and $m=0.1 \mathrm{day}^{-1}$. The elimination time of the source of the pollutant (the pulse width) is $t_{0}=120$ days. In the numerical calculations, the step lengths $\Delta x=\Delta y=0.1 \mathrm{~km}$ and $\Delta t=0.0005$ days have been used to achieve the stability of the finite difference scheme.

With the pollution source active $\left(t \leq t_{0}\right)$ and for a fixed retardation factor of $R_{f}=1.08$, Figure 1 shows the concentration profiles at various moments in time. These profiles are not in chronological order and the uppermost surface is for the middle value of time (90 days). The lower and middle surfaces are for the times of 70 and 110 days, respectively. This is due to the periodic nature of the input concentration. Near the source point $(x=1 \mathrm{~km}, y=1 \mathrm{~km})$, the distributions of the solute in the transverse and longitudinal directions are of similar order of magnitude (in terms of relative concentrations) despite the transverse component of the velocity and dispersion coefficient being merely one-tenth of the corresponding values for the longitudinal direction. One can observe at the far-end boundaries $x=0$ and $y=0$ that surfaces in Fig. 1 are flat. The concentration gradient with respect to time decreases.

For a parametric retardation factor of $R_{f}=1.08,1.38$ and 1.68, Figure 2 shows the concentration profiles at time $t=90$ days. Larger retardation factors indicate higher adsorption of the solute by the solid matrix of the porous medium. One can thus observe in Fig. 2 that the concentration decreases at a higher rate for higher values of this factor $R_{f}$.

Figures 3 and 4 show concentration profiles for time $t>t_{0}$. As the pollution source is then inactive (starting from $t_{0}=120$ days), the concentration at the source point ( $x=1 \mathrm{~km}$ and $y=1$ $\mathrm{km}$ ) becomes zero - after it had peaked in Fig. 1. For $t=140$, 160 and 180 days and for the retardation factor $R_{f}=1.08$ one can observe in Figure 3 that the peak concentration value decreases with time and drifts away from the point-source. Consequently, the peak concentration and its rate of change at a point can be on the rise after the pollution source is extinguished. The transverse transport speeds up the rehabilitation process. The trend of contaminant concentration with time and distance travelled is almost the same as for $t \leq t_{0}$. The concentration values are changing periodically with time and position. The upper surface represents solute concentration at $t=160$ days, while the middle and lower surfaces indicate lower and higher time at the same position.

Figure 4 shows the concentration profiles at time $t=160$ days, which is after the pollution source turned inactive $\left(t>t_{0}\right)$.

Results are shown for three values of the retardation factor: $R_{f}=1.08,1.38$ and 1.68. The solute concentration decreases with the increasing retardation factor. 


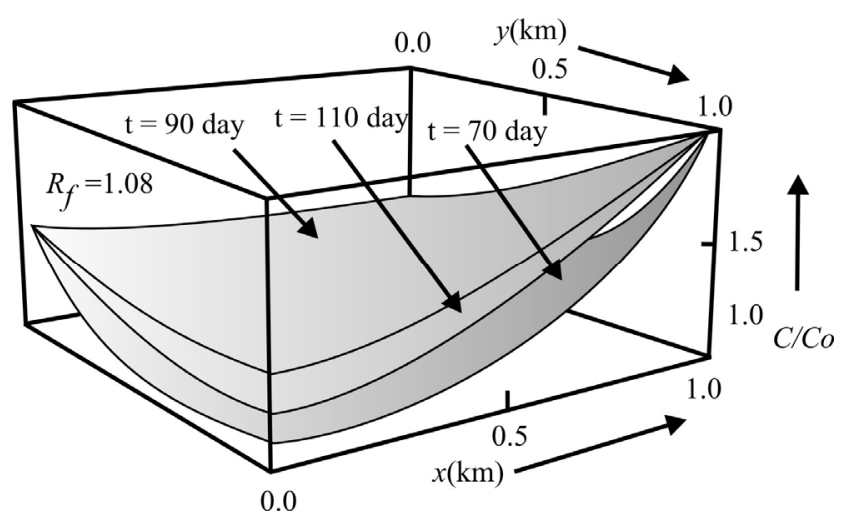

Fig. 1. Solute concentration in 2D space at time $t=70,90$ and 110 days (which is within the 120 days of the source activity), for a fixed retardation factor of $R_{f}=1.08$.

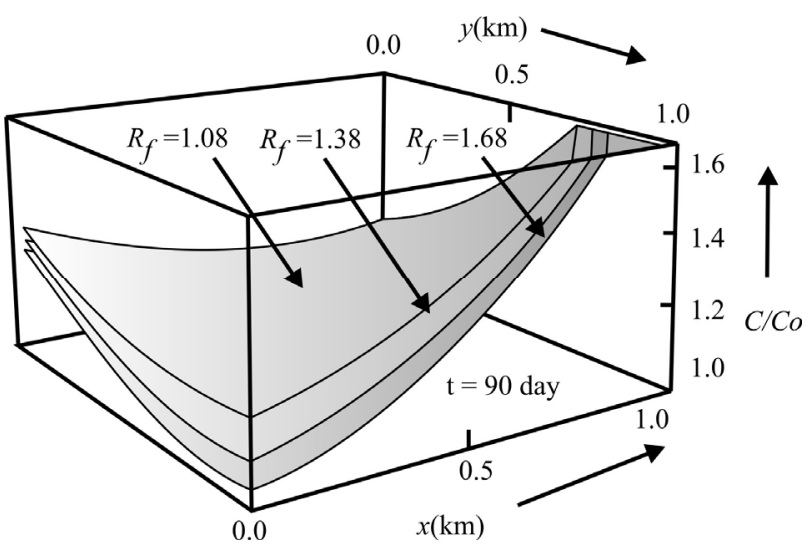

Fig. 2. Solute concentration in $2 \mathrm{D}$ space at time $t=90$ days (the source is active for 120 days) for a varied value of the retardation factor of $R_{f}=1.08,1.38$ and 1.68 .

While the numerical and analytical results match closely, a measure of this match is evaluated by the mean square error defined as:

error $=\frac{1}{N \cdot R} \sum_{i=1}^{N} \sum_{j=1}^{R}\left(C_{i, j}^{\text {num }}-C^{\text {analyt }}\right)^{2}$

where $N \cdot R$ is the total number of observation points. Values calculated by (18) are shown in Table 1 at $t=180$ days (the longest time analyzed), where it is assumed that the source was active for the first 120 days, and for a fixed retardation factor of $R_{f}=1.08$. The error values increase with time so the maximum deviation between the results obtained with analytical and numerical solutions over a 180 -day period is $\approx 0.001 \%$.

The second accuracy test was the time step value sensitivity. Due to the accuracy requirements of the EFDM, we used value $\Delta t=0.0005$ days for all EFDM runs. In contrast, as can be seen from Table 2, the EFDM method remains stable and accurate, even with a ten-times higher time step value.

Finally, the EFDM used in this work for solving ADE, besides it is the simplest among other FDMs (Dehghan, 2004), FEMs (Huang et al., (2008)) and FVMs (Gharehbaghi, (2017)), it is effective and accurate for solving time-dependent ADEs in twodimensional space. Stability of the explicit finite difference scheme proposed in this work and high accuracy of the obtained numerical results have easily been achieved by using a sufficiently small discrete time step length of $\Delta t=0.0005$ days.

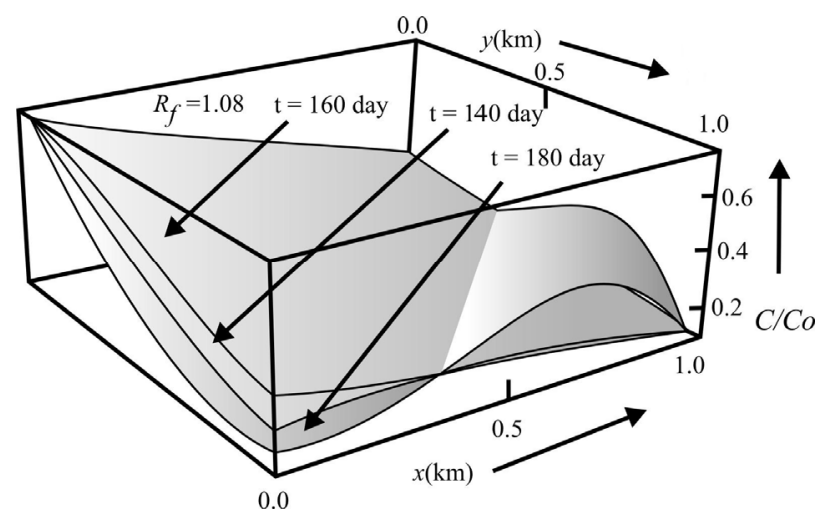

Fig. 3. Solute concentration in $2 \mathrm{D}$ space at time $t=140,160$ and 180 days (the source was active for the first 120 days), for a fixed retardation factor of $R_{f}=1.08$.

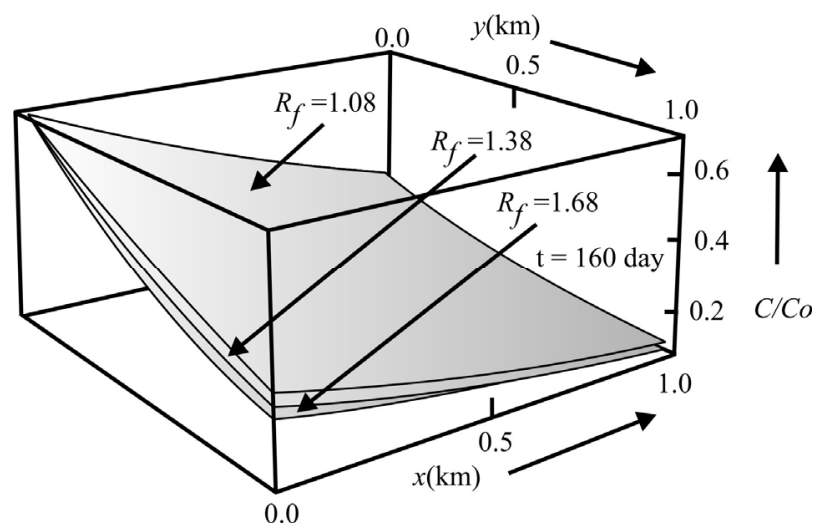

Fig. 4. Solute concentration in $2 \mathrm{D}$ space at time $t=160$ days (the source was active for the first 120 days), for a varied value of the retardation factor of $R_{f}=1.08,1.38$ and 1.68 .

Table 1. Errors in solute concentration shown in Fig. 3 at $t=20$ to $t=180$ days (the longest time analyzed); it is assumed that the source was active for the first 120 days and that the retardation factor was $R_{f}=1.08$.

\begin{tabular}{lc}
\hline$t$ (day) & error \\
\hline 20 & 0.00000237 \\
40 & 0.00000359 \\
60 & 0.00000475 \\
80 & 0.00000509 \\
100 & 0.00000641 \\
120 & 0.00000778 \\
140 & 0.00000905 \\
160 & 0.00001035 \\
180 & 0.00001159 \\
\hline
\end{tabular}

Table 2. Errors in solute concentration shown in Fig. 3 at $t=180$ days for various time steps $\Delta t$; it is assumed that the source was active for the first 120 days and that the retardation factor was $R_{f}=1.08$.

\begin{tabular}{lc}
\hline$\Delta t$ (day) & error \\
\hline 0.005 & 0.00001433 \\
0.002 & 0.00001332 \\
0.001 & 0.00001251 \\
0.0005 & 0.00001159 \\
\hline
\end{tabular}




\section{CONCLUSIONS}

A solution for the unsteady dispersion of pollution through a horizontal porous medium that is homogeneous using the explicit finite difference method is presented. Periodicity of velocity and boundary conditions simulate the transient advection-dispersion response of ground water systems to water level changes. Accounted for is the retardation of a solute that occurs in the porous media due to adsorption.

High accuracy of the method is apparent from the comparison of numerical results with those obtained with analytical solutions that are available for special cases. It has been achieved by setting sufficiently small discrete time step length of $\Delta t=0.0005$ days. The numerical solution was able to calculate the same solute transport scenario as expected and corresponded with the analytical solution. Furthermore, the EFDM presented in this work for solving ADE is the simplest among other FDMs, FEMs and FVMs. The method showed how the peak concentration changed with time and drifted away from the point source once this source became inactive. The significance of the transverse transport is apparent in speeding up the rehabilitation process. It was also found that the solute concentration reflects strongly the input periodicity of the seepage velocity.

Acknowledgment. The work described in this paper was supported by the Strategic Research Grant of City University of Hong Kong (Project No. CityU 7004600) and by the Serbian Ministry of Education and Science under Grant No. 171011.

\section{REFERENCES}

Ahmed, S.G., 2012. A numerical algorithm for solving advection-diffusion equation with constant and variable coefficients. The Open Num. Meth. J., 4, 1-7.

Al-Niami, A.N.S., Rushton, K.R., 1977. Analysis of flow against dispersion in porous media. J. Hydrol., 33, 87-97.

Anderson, J.D., 1995. Computational Fluid Dynamics, McGraw-Hill, New York.

Appadu, A.R., 2013. Numerical solution of the 1D advectiondiffusion equation using standard and nonstandard finite difference schemes, J. Appl. Math., 2013, 1-14.

Chatwin, P.C., Allen, C.M., 1985. Mathematical models of dispersion in rivers and estuaries. Ann. Rev. Fluid Mech., 17, 119-149.

Chaudhry, M.H., Cass, D.E., Edinger, J.E., 1983. Modelling of unsteady-flow water temperatures. J. Hydraul. Eng., 109, 5, 657-669.

Chen, J.-S. Liu, C.-W., 2011. Generalized analytical solution for advection-dispersion equation in finite spatial domain with arbitrary time-dependent inlet boundary condition Hydrol. Earth Syst. Sci., 15, 2471-2479.

Cherry, J.A., Gillham, R.W., Barker, J.F., 1984. Contaminants in Groundwater - Chemical Processes in Groundwater Contamination. National Academy Press, Washington, DC, pp. 46-64.

Ciftci, E., Avci, C.B., Borekci, O.S., Sahin, A.U., 2012. Assessment of advective-dispersive contaminant transport in heterogeneous aquifers using a meshless method. Environmental Earth Sci., 67, 2399-2409.

Dehghan, M., 2004. Weighted finite difference techniques for the one-dimensional advection-diffusion equation. Appl. Math. Computation, 147, 307-319.

Dhawan, S., Kapoor, S., Kumar, S., 2012. Numerical method for advection-diffusion equation using FEM and B-splines.
J. Computat. Sci., 3, 429-437.

Djordjevich, A., Savović, S., 2013. Solute transport with longitudinal and transverse diffusion in temporally and spatially dependent flow from a pulse type source. Int. J. Heat Mass Trans., 65, 321-326.

Fattah, Q.N., Hoopes, J.A., 1985. Dispersion in anisotropic homogeneous porous media. J. Hydraul. Eng., 111, 810827.

Gane, C.R., Stephenson, P.L., 1979. An explicit numerical method for solving transient combined heat conduction and convection problems. Int. J. Numer. Meth. Eng., 14, 11411163.

Gharehbaghi, A., 2016. Explicit and implicit forms of differential quadrature method for advection-diffusion equation with variable coefficients in semi-infinite domain. J. Hydrol., 504(B), 935-940.

Gharehbaghi, A., 2017. Third- and fifth-order finite volume schemes for advection-diffusion equation with variable coefficients in semi-infinite domain. Water Environm. J., 31, 2, 184-193.

Guvanasen, V., Volker, R.E., 1983. Numerical solutions for solute transport in unconfined aquifers. Int. J. Numer. Meth. Fluids, 3, 103-123.

Holly, F.M., Usseglio-Polatera, J.M., 1984. Dispersion simulation in two-dimensional tidal flow. J. Hydraul. Eng., 111, 905-926.

Huang, Q., Huang, G., Zhan, H., 2008. A finite element solution for the fractional advection-dispersion equation. Adv. Water Resour., 31, 1578-1589.

Jaiswal, D.K., Kumar, A., Kumar, N., Yadav, R.R., 2009. Analytical solutions for temporally and spatially dependent solute dispersion of pulse type input concentration in onedimensional semi-infinite medium. J. Hydro-Environm. Res., 2, 254-263.

Jaiswal, D.K., Kumar, A., Yadav, R.R., 2011. Analytical solution to the one-dimensional advection-diffusion equation with temporally dependent coefficients. J. Water Resource Protect., 3, 76-84.

Karahan, H., 2006. Implicit finite difference techniques for the advection-diffusion equation using spreadsheets. Adv. Eng. Software, 37, 601-608.

Kaya, B., Gharehbaghi, A., 2014. Implicit solutions of advection diffusion equation by various numerical methods. Aust. J. Basic \& Appl. Sci., 8, 1, 381-391.

Kumar, N., 1988. Unsteady flow against dispersion in finite porous media. J. Hydrol., 63, 345-358.

Kumar, A., Jaiswal, D.K., Kumar, N., 2010. Analytical solutions to one-dimensional advection-diffusion equation with variable coefficients in semi-infinite media. J. Hydrol., 380, 330-337.

Lapidus, L., Amundson, N.R., 1952. Mathematics of adsorption in beds, VI. The effects of longitudinal diffusion in ionexchange and chromatographic columns. J. Phys. Chem., 56, 8, 984-988.

Logan, J.D., Zlotnik, V., 1995. The convection-diffusion equation with periodic boundary conditions. Appl. Math. Lett., 8, 1995, 55-61.

Marino, M.A., 1978. Flow against dispersion in non-adsorbing porous media. J. Hydrol., 37, 149-158.

Nazir, T., Abbas, M., Ismail, A.I.M., Majid, A.A., Rashid, A., 2016. The numerical solution of advection-diffusion problems using new cubic trigonometric B-splines approach. Appl. Math. Modelling., 40, 7-8, 4586-4611.

Parlange, J.-Y., 1980. Water transport in soils. Ann. Rev. Fluid Mech., 12, 77-102. 
Salmon, J.R., Liggett, J.A., Gallager, R.H., 1980. Dispersion analysis in homogeneous lakes. Int. J. Numer. Meth. Eng., $15,1627-1642$.

Savović, S., Caldwell, J., 2003. Finite difference solution of one-dimensional Stefan problem with periodic boundary conditions. Int. J. Heat Mass Transfer, 46, 2911-2916.

Savović, S., Caldwell, J., 2009. Numerical solution of Stefan problem with time-dependent boundary conditions by variable space grid method. Thermal Sci., 13, 165-174.

Savović, S., Djordjevich, A., 2012. Finite difference solution of the one-dimensional advection-diffusion equation with variable coefficients in semi-infinite media. Int. J. Heat Mass Transfer, 55, 4291-4294.

Savović, S., Djordjevich, A., 2013. Numerical solution for temporally and spatially dependent solute dispersion of pulse type input concentration in semi-infinite media. Int. J. Heat Mass Transfer, 60, 291-295.

Siemieniuch, J.L., Gladwell, I., 1978. Analysis of explicit difference methods for a diffusion-convection equation. Int. J. Num. Meth. Eng., 12, 6, 899-916.

Šimůnek, J., van Genuchten, M.T., Šejna, M., Toride, N., Leij, F.J., 1999. The STANMOD computer software for evaluating solute transport in porous media using analytical solutions of convection-dispersion equation. Versions 1.0 and
2.0. IGWMC-TPS-71. Colorado School of Mines, International Ground Water Modeling Center, Golden, Colorado.

Townley, L.R., 1995. The response of aquifers to periodic forcing. Adv. Water Resour., 18, 125-146.

van Genuchten, M.T., Šimůnek, J., Leij, F.J., Toride, N., Šejna, M., 2012. STANMOD: Model use, calibration, and validation. Trans. ASABE, 55, 4, 1353-1366.

Yadav, R.R., Jaiswal, D.K., Yadav, H.K., Gulrana, 2011. Temporally dependent dispersion through semi-infinite homogeneous porous media: an analytical solution. Int. J. Res. Rev. Appl. Sc., 6, 158-164.

Yadav, R.R., Jaiswail, D.K., Gulrana, 2012. Two-dimensional solute transport for periodic flow in isotropic porous media: an analytical solution. Hydrol. Process., 26, 3425-3433.

Zhao, C., Valliappan, S., 1994. Numerical modelling of transient contaminant migration problems in infinite porous fractured media using finite/infinite element technique: theory. Int. J. Num. Anal. Meth. Geomech., 18, 523-541.

Zlatev, Z., Berkowicz, R., Prahm, L.P., 1984. Implementation of a variable stepsize variable formula in the timeintegration part of a code for treatment of long-range transport of air pollutants. J. Comput. Phys., 55, 278-301.

Received 24 March 2017 Accepted 1 August 2017 